\title{
Identification of a gene for an ancient cytokine, interleukin 15-like, in mammals; interleukins 2 and 15 co-evolved with this third family member, all sharing binding motifs for IL-15R $\alpha$
}

\author{
Johannes M. Dijkstra • Fumio Takizawa - Uwe Fischer • Maik Friedrich • \\ Veronica Soto-Lampe • Christophe Lefèvre • Matthias Lenk • Axel Karger • \\ Taei Matsui • Keiichiro Hashimoto
}

Received: 4 October 2013 / Accepted: 7 November 2013 / Published online: 26 November 2013

(C) The Author(s) 2013. This article is published with open access at Springerlink.com

\begin{abstract}
Interleukins 2 and 15 (IL-2 and IL-15) are highly differentiated but related cytokines with overlapping, yet also distinct functions, and established benefits for medical drug use. The present study identified a gene for an ancient third IL2/15 family member in reptiles and mammals, interleukin $15-$ like $(I L-15 L)$, which hitherto was only reported in fish. $I L-$ $15 \mathrm{~L}$ genes with intact open reading frames (ORFs) and evidence of transcription, and a recent past of purifying selection, were found for cattle, horse, sheep, pig and rabbit. In human and mouse the $I L-15 L$ ORF is incapacitated. Although deduced IL-15L proteins share only $\sim 21 \%$ overall amino acid identity with IL-15, they share many of the IL-15 residues
\end{abstract}

Electronic supplementary material The online version of this article (doi:10.1007/s00251-013-0747-0) contains supplementary material, which is available to authorized users.

J. M. Dijkstra $(\square) \cdot$ K. Hashimoto

Institute for Comprehensive Medical Science, Fujita Health

University, Dengakugakubo 1-98, Toyoake, Aichi 470-1192, Japan

e-mail: dijkstra@fujita-hu.ac.jp

F. Takizawa $\cdot$ U. Fischer $\cdot$ V. Soto-Lampe $\cdot$ M. Lenk Institute of Infectology, Friedrich-Loeffler-Institute, Boddenblick 5A, 17498 Insel Riems, Germany

M. Friedrich

Institute of Clinical Immunology, Max-Bürger Research Centre, Johannisallee 30, 04103 Leipzig, Germany

C. Lefèvre

Centre for Biotechnology and Interdisciplinary Sciences, Deakin

University, Pigdons Road, Geelong, Victoria 3217, Australia

A. Karger $\cdot$ T. Matsui

Institute of Molecular Biology, Friedrich-Loeffler-Institute,

Boddenblick 5A, 17498 Insel Riems, Germany important for binding to receptor chain IL- $15 \mathrm{R} \alpha$, and recombinant bovine IL-15L was shown to interact with IL-15R $\alpha$ indeed. Comparison of sequence motifs indicates that capacity for binding IL-15R $\alpha$ is an ancestral characteristic of the IL-2/ 15/15L family, in accordance with a recent study which showed that in fish both IL-2 and IL-15 can bind IL-15R $\alpha$. Evidence reveals that the species lineage leading to mammals started out with three similar cytokines IL-2, IL-15 and IL$15 \mathrm{~L}$, and that later in evolution (1) IL-2 and IL-2R $\alpha$ receptor chain acquired a new and specific binding mode and (2) IL$15 \mathrm{~L}$ was lost in several but not all groups of mammals. The present study forms an important step forward in understanding this potent family of cytokines, and may help to improve future strategies for their application in veterinarian and human medicine.

Keywords Cytokine - Evolution · Interleukins $2 \cdot 15$ and 15-like $\cdot$ Receptor

\section{Introduction}

A group of related short-chain helical cytokines IL-2, IL-4, IL7, IL-9, IL-15 and IL-21 bind receptors that have an IL-2R $\gamma$ chain (also known as "common cytokine-receptor $\gamma$-chain" or " $\gamma_{\mathrm{c}} "$ ) and play important roles in the immune system (Leonard 2008). Overall sequence similarity levels classify IL-2, IL-15 and IL-21 as a distinct subfamily (Parrish-Novak et al. 2002; Kono et al. 2008). Functional similarities indicate a close phylogenetic relationship between IL-2 and IL-15, because their respective receptor complexes IL-2R $\alpha \cdot \mathrm{IL}-2 \mathrm{R} \beta \cdot \mathrm{IL}-2 \mathrm{R} \gamma$ (Grabstein et al. 1994; Giri et al. 1994, 1995; Ring et al. 2012) 
and IL-15R $\alpha \cdot I L-2 R \beta \cdot I L-2 R \gamma$ (Grabstein et al. 1994; Giri et al. 1994, 1995; Ring et al. 2012) are unique by including IL-2R $\beta$ chain and a chain of the IL-2R $\alpha / 15 R \alpha$ family. The closely related IL-2R $\alpha$ and IL-15R $\alpha$ are encoded by tandemly duplicated genes (Anderson et al. 1995), are not related to other known cytokine receptor chains, and bind cytokines by their "sushi" domains (aliases "complement control protein" or "short consensus repeat" domains). The IL-2R $\alpha$ and IL$15 \mathrm{R} \alpha$ chains confer cytokine specificity, and affinities that are much higher than those of the IL-2R $\beta \cdot \mathrm{IL}-2 \mathrm{R} \gamma$ receptor alone (Sugamura et al. 1996; Giri et al. 1995; Waldmann and Tagaya 1999). The binding affinity of IL- 15 for IL- $15 R \alpha$ is exceptionally high $\left(K_{\mathrm{d}}=\sim 50 \mathrm{pM}\right.$; e.g., Mortier et al. 2006), and IL15 predominantly functions with co-expressed IL-15R $\alpha$ in either membrane-bound or released form as a stable heterodimer that can stimulate other cells which express IL-2R $\beta$.IL2R $\gamma$ (Dubois et al. 2002; Sandau et al. 2004; Mortier et al. 2008; Bergamaschi et al. 2008, 2012). This mode of presentation is called "trans-presentation", indicating that IL-15R $\alpha$ is not expressed by the same cell as IL-2R $\beta \cdot \mathrm{IL}-2 \mathrm{R} \gamma$. The binding affinity of IL-2 for IL-2R $\alpha$ is much lower $\left(K_{\mathrm{d}}=\sim 20 \mathrm{nM}\right.$; e.g., Myszka et al. 1996), and IL-2R $\alpha$ tends to function within co-expressed IL-2R $\alpha \cdot I L-2 R \beta \cdot I L-2 R \gamma$ complexes that have the three receptor chains all inserted in the same membrane (cis-presentation) and can bind free secreted IL-2. Signaling through IL-2 and IL-15 receptors is mediated intracellularly by the cytoplasmic tails of IL-2R $\beta$ and IL-2R $\gamma$.

IL-2 protein is predominantly expressed by activated T cells (Taniguchi et al. 1983; Malek 2008), whereas dendritic cells and monocytes are important for expression of IL-15 protein (Waldmann 2006). In vitro assays show substantial overlap in IL-2 and IL-15 functions involving survival, proliferation and differentiation of various $\mathrm{B}, \mathrm{T}$ and natural killer (NK) cell populations (Taniguchi et al. 1983; Grabstein et al. 1994; Waldmann 2006; Malek 2008; Ring et al. 2012). Pronounced differences between IL-2 and IL-15 functions, however, are apparent when comparing genetically engineered mice. IL-15-deficient mice show marked defects in the production/maintenance of NK cells, natural killer T (NKT) cells, intestinal intraepithelial lymphocytes, and CD8 memory cells (Kennedy et al. 2000; Waldmann 2006). In contrast, IL-2-deficient mice show lymphoproliferative and autoimmune disorder, caused by a defect in the production of $\mathrm{CD} 4^{+} \mathrm{CD} 25^{+} \mathrm{Foxp}^{+} \mathrm{T}$ regulatory (Treg) cells (Sadlack et al. 1995; Almeida et al. 2002; Malek 2008). The sensitivity of Treg cells to IL-2 correlates with their constitutive expression of high levels of IL-2R $\alpha$ (alias CD25; Fontenot et al. 2005; Malek 2008).

Recombinant IL-2 has been established as an anti-cancer drug (Waldmann 2006), but because of the dual function of both enhancing and downregulating immune responses, modified IL-2 with less specificity for Treg has been developed
(Levin et al. 2012; Liao et al. 2013). IL-15 lacks this duality and may be a more promising anti-cancer agent than IL-2, especially if its stability and potency are enhanced by recombinant combination with IL-15R $\alpha$ (Mortier et al. 2006; Vincent et al. 2013). Blockage of IL-2R $\alpha$ or IL-15R $\alpha$ function is medically used, or investigated for that purpose, to halt lymphoma progression or inflammation (Waldmann 2006; Wang et al. 2010).

The sequences of short-chain type I helical cytokines are very poorly conserved, even among orthologues (Huising et al. 2006), which is exemplified by the initial inability to properly distinguish between $I L-2$ and $I L-15$ identity of chicken $I L-2$ (Sundick and GillDixon 1997; Choi et al. 1999). More recently, however, the availability of whole genome sequences allowed reliable identification of $I L-2$ and $I L-15$ in various tetrapod species and teleost fishes because of gene synteny arguments (Kaiser and Mariani 1999; Bird et al. 2005; Bei et al. 2006; Fang et al. 2006; Gunimaladevi et al. 2007; Wang et al. 2007; Ohtani et al. 2008).

In teleost fish, a gene for an additional IL-2/15 family member was found which was designated IL-15-like (IL15L; Bei et al. 2006; Gunimaladevi et al. 2007), alias IL-15x (Fang et al. 2006). The function of fish $I L-15 L$ was not determined. The present study is the first to identify $I L-15 L$ genes and transcripts in mammals, to carefully analyze deduced IL-15L molecular features, and to describe interaction of recombinant IL-15L with IL- $15 \mathrm{R} \alpha$. It also comprises the first thorough analysis of IL-2 versus IL-15 sequence evolution.

\section{Results and discussion}

Identification of IL-15L in genome sequences of reptiles and mammals

Probably because of its pseudogene nature in human and mouse, $I L-15 L$ has not been reported outside fish. However, after scrutinizing available genome sequence databases for vertebrate species, we here present $I L-15 L$ gene in reptiles and mammals, which as in fish maps between the genes PLEKHG2 and SUPT5H (Fig. S1A). In tetrapod $I L-15 L$ the family consensus intron between exons 3 and 4 was lost, without hampering the coding capacity, and the resulting larger exon is referred to in this article as "exon 3/4" (Fig. S1B ). In birds or amphibians $I L-15 L$ could not be found, despite extensive searches, and the gene may have been lost in these animal classes. The cladogram in Fig. 1 shows the distribution among species of $I L-15 L$, and distinguishes between consensus intact open reading frames (ORFs) (white circles), non-typical but possibly intact ORFs (gray circles), and incapacitated ORFs (black circles); half circles refer to incomplete sequence information (for details, see Fig. S2). 


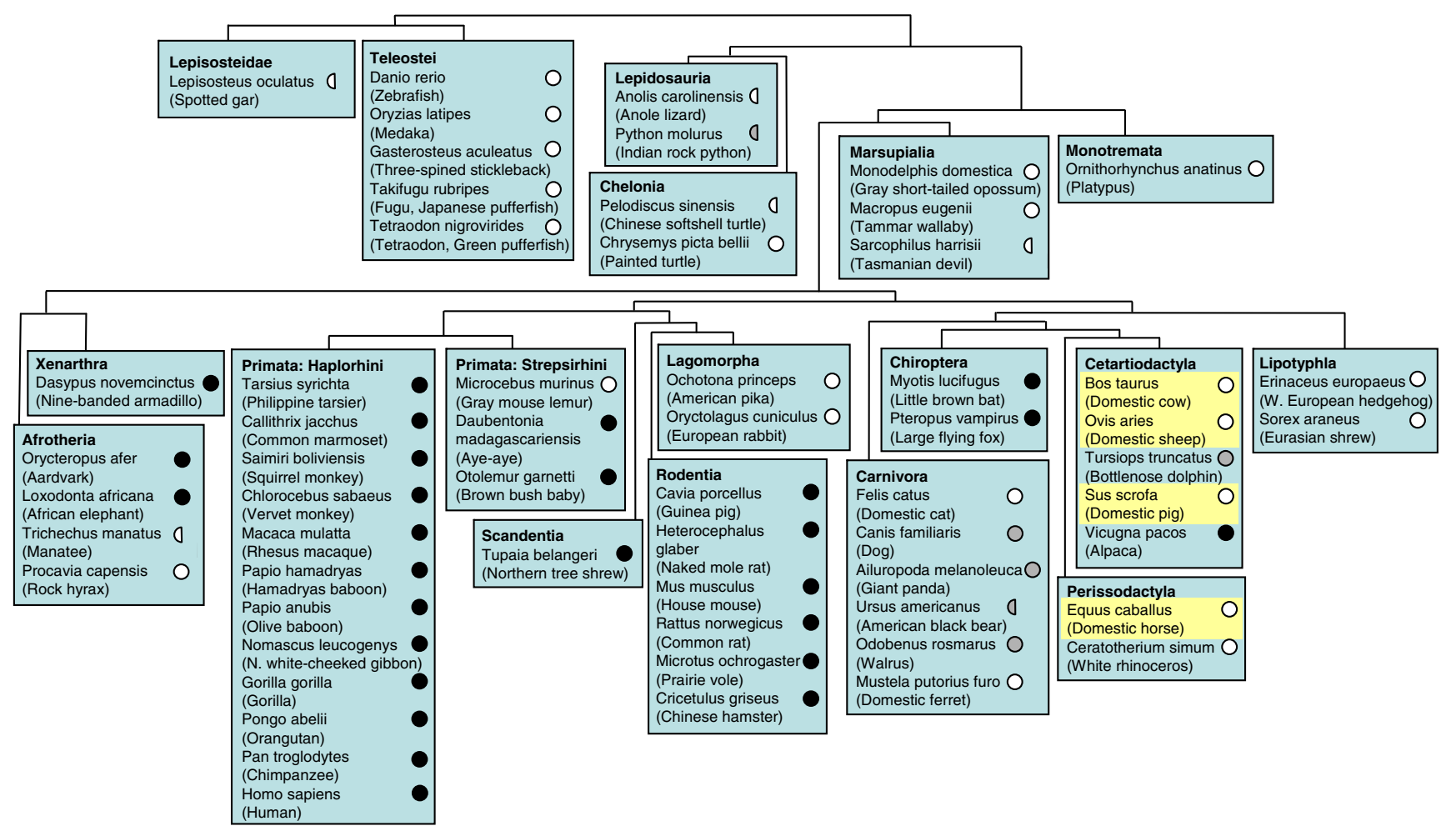

Fig. 1 Phylogenetic distribution of $I L-15 L$. White circles represent consensus intact $I L-15 L$ ORFs, gray circles represent non-typical but possibly intact $I L-15 L$ ORFs, and black circles represent incapacitated $I L-15 L$

Intact $I L-15 L$ may be common in reptiles and non-eutherian mammals (monotremes plus marsupials), while in many eutherian mammals the ORF was incapacitated (Fig. 1 and Fig. S2). In eutherian mammals intact $I L-15 L$ ORF could be found in rock hyrax, gray mouse lemur, rabbit, pika, cat, ferret, horse, rhinoceros, cattle, sheep, pig, hedgehog, and shrew (Fig. 1 and Fig. S2), which interestingly include the four most important agricultural mammals (highlighted in yellow in Fig. 1). Database sequences may contain errors, and at the individual species level the detected ORF incapacitation motifs may not always represent the biological situation. However, by comparison of related species, such as for example among primates, some of the incapacitation motifs could be confirmed in independent databases (Fig. S2). In the human genome, large parts of incapacitated $I L-15 L$ remain, while in mouse only minor remnants are found (Fig. S2). Despite some modifications, the $I L-2, I L-15, I L-15 L$ and $I L-21$ loci are relatively well conserved throughout classes of jawed vertebrates (Fig. S1A), which may be rather common among genes of cytokines involved in $\mathrm{T}$ cell differentiation (Secombes et al. 2011). The relatively well conserved nature of $I L-2 / 15 / 15 L / 21$ family loci contrasts the evolution pattern of other families of secreted mediators of the immune system such as chemokines and type I interferons, which experienced more extensive gene expansions, contractions and translocations (Nomiyama et al. 2008; Xu et al. 2013).
ORFs; half circles refer to incomplete sequence information (for details, see Fig. S2). Mammals important for agriculture are highlighted in yellow. Branch knots within mammals are based on Meredith et al. (2011)

Independent $I L-15 L$ ORF incapacitations in different lineages of eutherian mammals (Fig. 1 and Fig. S2) suggest that after the separation from marsupials the IL-15L protein considerably lost in importance early in the eutherian mammal clade. However, lack of importance in one animal does not necessarily conclude the same for others. The eutherian mammal superorder in which IL-15L may have retained a relatively high importance, as indicated by the distribution of intact $I L-15 L$ ORFs, is Laurasiatheria, the superorder which includes Carnivora, Chiroptera, Cetartiodactyla, Perissodactyla, and Lipotyphla (Fig. 1). In some Cetartiodactyla and Perissodactyla like cattle, sheep, horse and rhinoceros, the potential importance at the protein level, which may have been retained, regained or newly acquired, is underlined by modifications around the start codon which are predicted to ensure a higher efficiency of translation (Fig. S2).

Analysis of IL-15L transcripts in mammals

Evidence for IL-15L coding transcripts in cattle, horse, pig and sheep, as well as in rabbit, could be obtained (Figs. S3 and S5). The major types of transcripts that we found are schematically summarized in Fig. 2, with Fig. 2a showing a typical transcript that encodes intact IL-15L. Bovine and rabbit $I L$ $15 \mathrm{~L}$ transcripts were investigated extensively, including $5^{\prime}$ RACE and 3'RACE analyses (Fig. S3A and $C$ ). Bovine and 
A

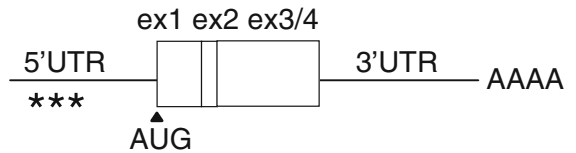

B

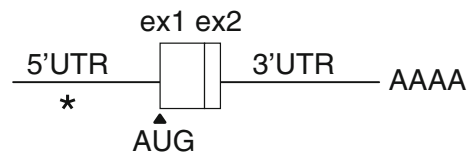

C

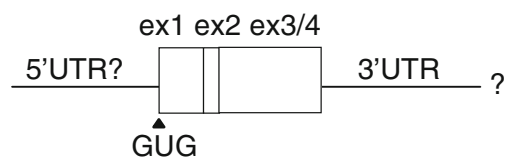

D

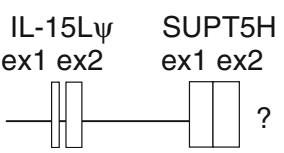

Fig. 2 Schematic view of different types of mammalian $I L-15 L$ transcripts. a Transcript with consensus $I L-15 L$ ORF and additional out of frame AUGs in the 5'UTR (asterisks). Full-length transcripts including poly-A tail (AAAA) were determined for cattle and rabbit (Fig. S3). b In rabbits, however, most transcripts lack the exon $3 / 4$ sequence (ex3/4) and are unlikely to encode IL-15L protein (Figs. S3C-E and $\mathrm{S} 5 B$, middle picture). $\mathbf{c}$ In dog and bear, the consensus $I L-15 L$ AUG start codon has been replaced by GUG, and the region 5 ' thereof is unlikely to encode the $\mathrm{N}$-terminus of a functional cytokine (Figs. S2 and $\mathrm{S} 4 A-C$ ). It was not determined whether these transcripts have a poly-A tail. d For human a hybrid $I L-15 L \psi-S U P T 5 H$ cDNA sequence was reported (GenBank DC400386), which is unlikely to encode modified IL-15L protein (Fig. S4D and $E$ ). We did not investigate the $5^{\prime}$ and $3^{\prime}$ ends of this type of transcript

rabbit $I L-15 L$ transcripts could be amplified from all investigated tissues and from various fibroblastoid and epithelioid permanent cell lines, and the results suggest low but ubiquitous expression (Fig. S5). A higher expression of $I L-15 L$ transcripts in organs of the immune system such as thymus, lymph nodes, or spleen, was not observed (Fig. S5), similar to previous findings in fish (Bei et al. 2006; Gunimaladevi et al. 2007). That mammalian $I L-15 L$ expression is generally low was not only indicated by the large number of PCR cycles necessary for detection (Fig. S5; and by our initial difficulties to establish positive RT-PCR conditions), but also by the near absence of $I L-15 L$ sequences in EST databases (see below).

In the 5'UTR of $I L-15 L$ transcripts additional out-of-frame AUG codons can be found (Fig. 2, asterisks), namely, three or six in cattle (Fig. S3A and $B$ ) and one in rabbit (Fig. S $C$ and $D)$. Such upstream AUGs tend to interfere negatively with the efficiency of translation and are abundant in $I L-15$ where they are believed to be important in translational control (Waldmann and Tagaya 1999).

Not all mammalian $I L-15 L$ transcripts have apparent protein coding capacity. In rabbit, most transcripts do not contain exon $3 / 4$ sequence, but are organized as schematically shown in Fig. $2 b$ and are not expected to encode functional protein
(Fig. S3C-E). And in some species, like dogs and bears, the $I L-15 L$ consensus start codon has been replaced by GUG, which is schematically shown in Fig. 2c (details for these carnivores are shown in Fig. S $4 A-C$ ). Furthermore, as depicted in Fig. 2d, a human cDNA sequence reported in the EST database (GenBank DC400386) contains both an $I L$ $15 L \psi$ part and a part of the downstream SUPT5H gene (details in Fig. S4D and $E$ ). This human $I L-15 L \psi$-SUPT5H sequence and an American black bear EST sequence (GenBank GW294330; Fig. S4B), which both presumably do not encode (modified) IL-15L protein (Fig. S4B and $E$ ), are the only two mammalian $I L-15 L$ sequences in the NCBI EST database with evidence of transcription (intron sequences were spliced out). Transcripts that may not encode protein are not unique to mammalian $I L-15 L$, since splice variants with no apparent protein coding function were also described for teleost fish $I L-15 L$ (Gunimaladevi et al. 2007) and mammalian $I L-15$ is well known for its ubiquitous transcripts compared to a much more restricted protein distribution (Waldmann and Tagaya 1999).

Somewhat reminiscent of the $I L-15 L$ story is that of interleukin $26(I L-26)$. Although $I L-26$ intact gene and IL-26 protein function were described for humans (Donnelly et al. 2010), IL-26 ORF incapacitations were observed in several independent mammalian lineages, and multiple $I L-26$ transcripts with unknown function were found in species with incapacitated ORF (Shakhsi-Niaei et al. 2013).

Purifying selection on coding capacity of intact mammalian IL-15L genes

Comparison between the intact $I L-15 L$ ORFs found in various mammalian species indicates high rates of synonymous versus non-synonymous nucleotide substitutions with an average $d_{\mathrm{S}} / d_{\mathrm{n}}$ value of 5.3 (Table S2A). This reveals purifying selection after these animals separated in time, and suggests protein function in the extant species. Similar analyses for $I L$ 15 (Table S2B) and $I L-2$ (Table S2C) in the nearly same sets of mammalian species calculate $\mathrm{d}_{\mathrm{s}} / \mathrm{d}_{\mathrm{n}}$ values of only 3.8 and 2.8 , respectively, which indicates that sequence conservation pressure on IL-15L is relatively high compared to its family members. At the protein level, the absolute degrees of conservation appear similar within IL-15 and IL-15L evolution, which in mammals are clearly higher than found for IL-2 (compare $d_{\mathrm{n}}$ values in Table $\mathrm{S} 2$, but also see Fig. 3 and Fig. S6 $A$ and $C$ ).

Analysis of deduced amino acid sequences; IL-15L is similar to IL-2 and IL-15

Figure 3 compares amino acid sequences deduced from partially reconstituted human $I L-15 L$ pseudogene (human IL- 
shading of contributing cysteines. A deduced ancestral motif that can be found in many IL-2, IL-15, IL-15L and IL-21 molecules, while distinguishing them from other short-chain helical cytokines, consists of two cysteine pairs (Fig. 3, yellow and pink C's), an LXTP motif (residues 27-30), F44, D/E89 and Q108 (Fig. 3 and Fig. S6 $A$; residue numbering throughout this article agrees with numbers above these alignments). The IL-15L molecules have an additional cysteine pair (Fig. 3, green C's) which may connect the $\mathrm{N}$ - and C-terminal regions by disulfide bridge as found in human IL-4 (magenta C's); a similar bridge may also be present in avian IL-15 (orange C's). The residues Y28, E47 and D/E54 are well conserved between IL-2, IL-15 and IL-15L, but are absent in most IL-21, and these are exactly the key residues for binding of IL-15 to IL$15 \mathrm{R} \alpha$ (Olsen et al. 2007; see below). This agrees with IL-21 not binding a sushi-domain containing receptor (Leonard 2008). It is notable that only after the ancestors of mammals and birds separated, IL-2 in the mammalian lineage differentiated from family consensus by losing a cysteine pair (Fig. 3, yellow C's), by getting a longer exon2, and by losing the above-mentioned D/E54 residue (Fig. 3 and Fig. S6A).

Phylogeny within the IL-2/15/15L family; IL-2 and IL-15 may be each other's closest relatives

The members of the short chain helix cytokine family rapidly diverged, and even cysteine bridges and helix lengths are not well conserved (Rozwarski et al. 1994). The ancientness of the phylogenetic issues in question, the evolutionary rapidness of the changes, the plasticity of the sequences and molecule structures, and also the shortness of this family of molecules, make it difficult for specialized software programs to resolve their phylogenetic relationships (Fig. S6B; e.g., Gunimaladevi et al. 2007; Kono et al. 2008), because of the following reasons: (1) "chance occurrence" — rather than homogenous gradual diversification - played a relatively large role in how much the molecules diverged from the original; (2) various amino acid positions may be "saturated" from calculation perspective (Van de Peer et al. 2002); (3) alignments remain questionable. Therefore, for the distinction of IL-2/15/15L/21 and IL-2/15/15L as true phylogenetic (sub-)families, we believe that better evidence is provided by the distribution of "hallmark" motifs such as discussed in the above paragraph. Furthermore, percentages of identical amino acids also support the existence of the IL-2/15/15L family as a true phylogenetic group (Fig. S6C). The similarity comparisons in Fig. S6C underline that mammalian IL-2 differentiated to a relatively high degree from family consensus, an observation made as well for IL-2 in turtles (Fig. S6C) which similar to mammalian IL-2 did not retain all family consensus cysteine pairs (Fig. S6A).
Our alignments of the highly differentiated $\alpha$-helices A and C (Fig. 3 and Fig. S6A) remain discussable between different cytokines, but in some instances also between sequences of the same cytokine in different species. Nevertheless, for speculation on phylogeny within the IL-2/15/15L family it may be useful to look at the D11+N65 motif which in both mammalian IL-2 and IL-15 is important for binding IL-2R $\beta$ (e.g., Wang et al. 2005; Ring et al. 2012), and which is not found in IL-15L (diamonds in Fig. 3 and Fig. S6A). IL-15L may share an evolutionary older motif for type I receptor binding, namely, E/Q7+R68, with IL-21 and IL-4 (Hage et al. 1999; Bondensgaard et al. 2007; Hamming et al. 2012; inverted solid triangles in Fig. 3 and Fig. S6A). The Q107 residue important for binding IL-2R $\gamma$ found in IL-2, IL-15 and IL-21 (Wang et al. 2005; Ring et al. 2012; Hamming et al. 2012) is highly conserved in IL-15L (inverted open triangle in Fig. 3 and Fig. S6A), and we speculate that IL-15L can bind one of the type I receptors IL-2R $\beta \cdot \mathrm{IL}-2 \mathrm{R} \gamma$ or IL-21R $\alpha \cdot \mathrm{IL}-2 \mathrm{R} \gamma$ in a similar manner as by which IL-4 and IL-21 bind their respective type I receptors, which roughly includes one turn helical shifts of the cytokine helices $\mathrm{A}$ and $\mathrm{C}$ over the receptor binding site if compared with IL-2 and IL-15 (Bondensgaard et al. 2007; Hamming et al. 2012). If the mode by which IL-2 and IL-15 bind their type I receptor is unique and different from IL-15L indeed, that would suggest that IL-2 and IL-15 are each other's closest relatives. Future structural analyses of the type I receptor binding modes of IL-2/15/15L family members other than mammalian IL-2 and IL-15 should be helpful to clarify this matter.

Conservation of binding motifs for IL-15R $\alpha$

in the IL-2/15/15L family; recombinant IL-15L interacts with IL-15R $\alpha$

Olsen et al. (2007) distinguished the two regions of the mammalian IL-15.IL-15R $\alpha$ interface as "patch 1" and "patch 2", and in Fig. 3 the IL-15 residues contributing to these interactions are indicated with "1" and "2" below and the residues are shaded blue and pink, respectively. Identical residues in the other aligned sequences are shaded equally, and the most important key residues for IL-15R $\alpha$ interaction (Olsen et al. 2007) are indicated by circles above the alignment. Although the IL-15·IL-15R $\alpha$ interface patches 1 and 2 spatially roughly correspond with the two regions of the mammalian IL-2-IL$2 \mathrm{R} \alpha$ interface (Fig. 3, contributing IL-2 residues are indicated with "1" and "2" below), only the patch 2 residues in mammalian IL-2 resemble those of IL-15 (Rickert et al. 2005; Wang et al. 2005; Stauber et al. 2006; Chirifu et al. 2007; Olsen et al. 2007; Ring et al. 2012). Whereas the dominant patch 1 interactions are charged in IL-15.IL-15R $\alpha$, they are hydrophobic in mammalian IL-2.IL-2R $\alpha$, with a prominent role for IL-2 residue F25 (Rickert et al. 2005; Olsen et al. 2007). Figure 3 shows that only in mammalian IL-2 the patch 
1 residues markedly differ from IL-2/15/15L family consensus, and indeed it was found that in teleost fish both IL-2 and IL-15 can bind IL-15R $\alpha$ (Wen et al. 2011).

As expected from the sequence alignment (Fig. 3 and Fig. S6A), IL-15L could be shown to interact with IL-15R $\alpha$ in experiments using mammalian cells transfected with DNA plasmid expression vectors (Fig. S7A). In particular, recombinant bovine IL-15L was found on the surface of transfected cells only in the presence of recombinant bovine IL-15R $\alpha$ and not in the presence of recombinant bovine IL-2R $\alpha$ or absence of recombinant receptor (Fig. 4, upper left histograms), while the IL-15L molecules could be found intracellularly in all three experiments (Fig. 4, lower left histograms). Furthermore, recombinant co-expression with soluble bovine IL-15R $\alpha$ resulted in detectable IL-15L in the cell supernatant, whereas recombinant expression of IL-15L alone did not (Fig. S7B). These experiments (Fig. 4 and Fig. S7) suggest that bovine IL-15L depends on interaction with IL-15R $\alpha$ for efficient transport to and/or stability in the extracellular space, reminiscent of previous findings for IL-15 (Bergamaschi et al. 2008, 2012).

Remarkable conservation throughout jawed vertebrates of the IL-15R $\alpha$ motif for cytokine binding

Olsen et al. (2007) concluded that important IL-15R $\alpha$ residues for binding IL-15 were well conserved between birds and mammals. Fang et al. (2007) already reported $I L-15 R \alpha$ in teleost fish, and in the present study our database searches identified $I L-15 R \alpha$ sequences in gar and elephant shark, which are primitive bony and cartilaginous fishes, respectively. Sequence comparisons show that also in IL-15R $\alpha$ of fishes the motif for IL-15 binding is very well conserved (Fig. 5). In Fig. 5, the single-exon encoded major part of the sushi domain of IL- $15 R \alpha$ is aligned with corresponding sequences of the first sushi domain of IL-2R $\alpha$ in representative animals; these sequence fragments contain most of the cytokine binding
Fig. 4 Surface presentation of IL-15L by IL- $15 R \alpha$. The expression of bovine IL-15LFLAG, bovine IL-2R $\alpha$-Myc-His and bovine IL-15R $\alpha$-Myc-His in transfected HEK-293 cells was assayed by flow cytometry using anti-FLAG (left) and anti-Myc (right) mAbs. Surface binding was monitored using live cells (above) and transfection efficiencies were monitored using fixed/permeabilized cells (below). Only in the presence of IL- $15 \mathrm{R} \alpha$ receptor chain, IL-15LFLAG could be observed at the cell surface by using anti-FLAG (upper left histogram). Because the Myc-tags of IL-2R $\alpha$ and IL- $15 R \alpha$ were connected to the intracellular cytoplasmic tails, anti-Myc fluorescence could only be observed in fixed/ permeabilized cells. Percentages refer to the cells within the fluorescence range indicated by horizontal bar. The data shown are of a single experiment, representative of three independent experiments
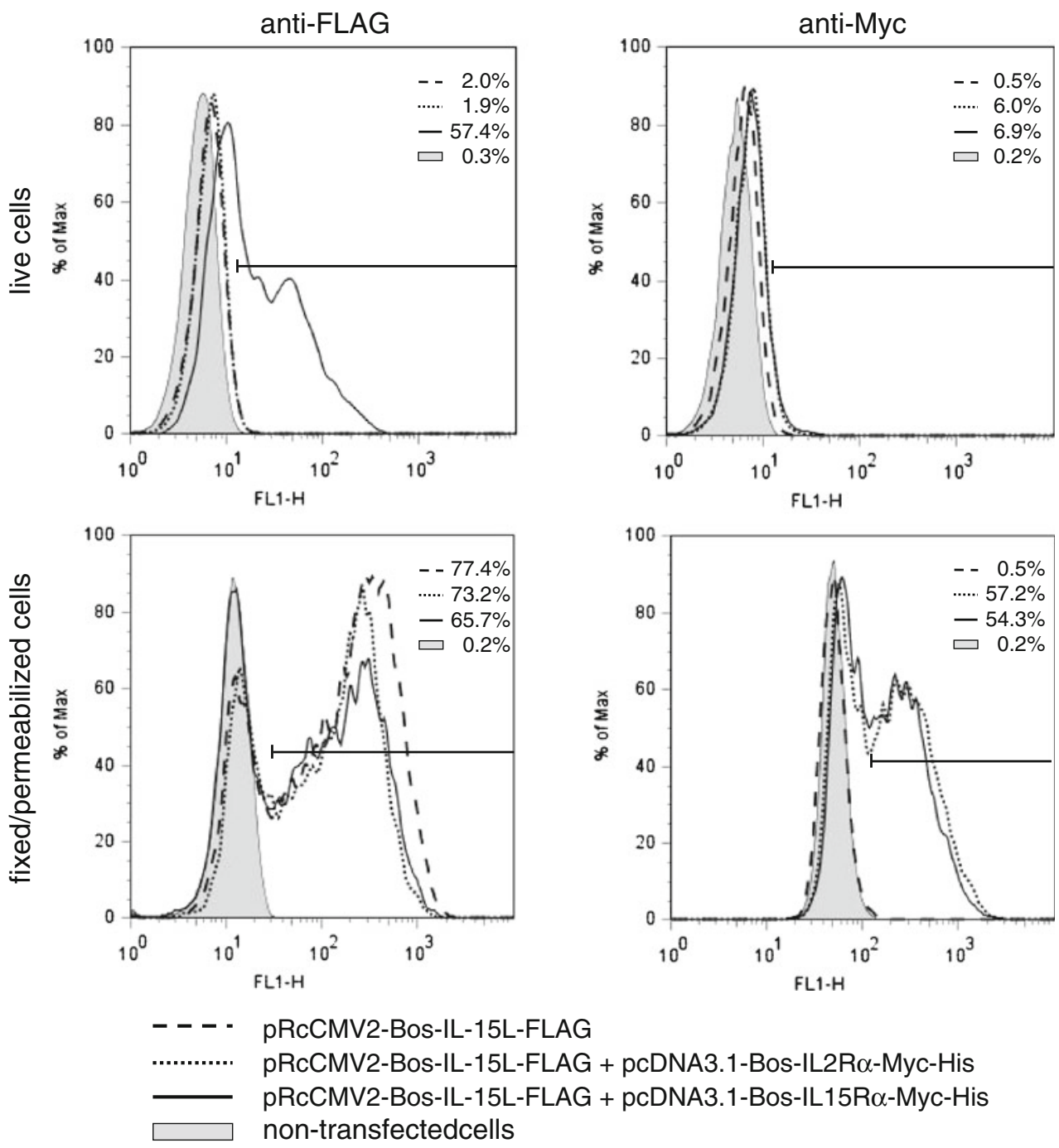
Human IL-15Ra

Mouse IL-15Ra

Cattle IL-15Ra

Opossum IL-15Ra

Chicken IL-15Ra

Lizard IL-15Ra

Frog IL-15Ra

Gar IL-15Ra

Pufferfish IL-15Ra

Elephant shark IL-15Ra

Frog IL-2Ra

Lizard IL-2Ra

Chicken IL-2Ra

Platypus IL-2Ra

Opossum IL-2Ra

Cattle IL-2Ra

Mouse IL-2Ra

Human IL-2Ra
ITCPPPM---SVEHA--DIWVKSYSLYSRE $\overline{\mathbf{R}} Y$ ICNSGFK $\overline{\mathbf{R}} K \overline{\mathbf{A}}$ GTSSLTECVLNKATNVAHWTT-PSI KCT TTCPPPV---SIEHA--DIRVKNYSVNSRERYVCNSGFKRKAGTSTLIECVINKNTNVAHWTT-PSLKCI ITCPTPT---SVEHA--DIQVKSYS INSRERYVCNSGEKRKAGTSSLTQCVFNETAKVAHWTT-PNLKCI TCPTPR---SVEHA--DIQVKSYNVNSRERYVCHSGFKRKAGTSSLTECI LEQNSNIAHWTE-PNLKCI VQCGRPK---DVANAI-INADDTALVNTILRYTCKLGYKRKAGTSTLIQCIL--ISDKPVWTH-TELQCI ECGVPT---LVENAQ----IAEGSNSEKLRYSCMKNYKRKAGT SNLILCKQDKHTKEYQWTN-SNLICI CSTPK---KVEHAN--DIEGEYKLDSVVRYKCQTDYKREAGT SNLAVCML--IDGKAQWSY-GNISCI YCSPPAPLSDTEHPN--LARR-FNEGAVFRYNCTVGYVRKAGTSNLIRCV--KKNNTLVWSQ-SNLICI ESACPCPKI--PPVNLTEAPPTDCFQIDAKFRYKCKAGYVRKAGRSNLITCIL--KGHRAEWTQ-PDLLCI SCGRPDLNVPNVNMS-VITKNSFKVGDRLRLTCDNGYVRKAGT SNLIHC----EANQFQWSK-PNLTCI CLYHF---PEDIII--I PEQQFFLGT I INLSCNKEYQRERNTHGMYTCV--NNSGVLKWKTEKDLKCI ECPFPQ---TIEFAE--YFAEWYVLGTVVRYDCELGYKRLGGRSNRMTCE--KRSTQAQWTCRFPPNCT KCPRLS---TTEFAD--VAAETYPLKTKLRYECDSGYRRRSGNTLTIRCQ--NVSGTASWVH-DELVCI KCPPPN---KI PFAK--YTAESYLKGSOLAYRCNKGYIRROGSEHFIECK--NHSGHLEWNT-FECFPT ECPHPP---QIDFAS--FATQIYMKGTFLECSCKPGYDRREGTPLFIVCEA--KDQHLSWS--EKCQCKVKS ACHDDPP--SLRNAM--FKVFRYEVGTMINCDCKTGFRRVSA---VMRCVG--DSSHSAWE--NRCFCNST LCLYDPP--EVPNAT--FKALSYKNGT I LNCECKRGFRRLKE-LVYMRCLG-------NSWS--SNCOCTSN LCDDDPP--EIPHAT--FKAMAYKEGTMLNCECKRGFRRIKSGSLYMLCTG--NSSHSSWD--NQCQCTSS \#\#\# \#

Fig. 5 Alignment of major parts of the sushi-domain of IL-15R $\alpha$ and the first sushi-domain of IL-2R $\alpha$ in representative species. All depicted fragments are encoded by a single exon. The numbers 1 and 2 above and below the alignment indicate the human IL-15R $\alpha$ and IL-2R $\alpha$ residues, which interact with IL-15 and IL-2 in patches 1 and 2, respectively, with the important key residues being underlined (Rickert et al. 2005; Olsen et al. 2007). To highlight the evolutionary conservation of the IL- $15 \mathrm{R} \alpha$ residues contributing to patches 1 and 2, these residues are shaded blue and pink throughout the alignment, including in IL-2R $\alpha$. Other human IL-2R $\alpha$ residues contributing to binding patches 1 and 2, or

residues. To our knowledge, Fig. 5 constitutes the first analysis of the evolution of the cytokine binding motif of IL-2R $\alpha$. In fish no $I L-2 R \alpha$ sequences could be found (see also Wen et al. 2011), and it is unclear whether in bony fish $I L-2 R \alpha$ gene was lost or that $I L-2 R \alpha$ was only established in the tetrapod line (Fig. S1C-F).

In Fig. 5, the human IL-15R $\alpha$ residues for binding IL-15 are colored blue and pink for patches 1 and 2, respectively, as was done for identical matching residues of the other sequences in the alignment. The well conserved IL-15R $\alpha$ motif for binding IL-15 is not known in other sushi-domain molecules (http://smart.embl.de) and hence at the level of cartilaginous fish already an IL-2/15/15L family member with high affinity for IL-15R $\alpha$ may have established, although we have not found a matching cytokine gene yet in this group of species for which sequence databases are incomplete. In databases of animals more primitive than jawed vertebrates, we could not find genes for IL-2/15/15L family cytokines or for IL- $2 R \alpha / 15 R \alpha$ receptors. In comparison with IL- $15 R \alpha$ to IL15 binding, the IL-2R $\alpha$ residues for binding IL-2 are conserved rather poorly between species (Fig. 5), which agrees with the diversification of the IL-2 sequences (Fig. 3 and Fig. S6) and the relative weakness of the binding between IL-2R $\alpha$ and IL-2. The fact that the binding motif for IL-15R $\alpha$ is well conserved in IL-15L suggests that this cytokine binds IL$15 \mathrm{R} \alpha$ with high affinity, but that remains to be determined experimentally.

Conservation of residue E47 throughout the IL-2/15/15L cytokines (Fig. 3 and Fig. S6A) and residue R35 throughout binding to IL-2 residues contributing to those patches, are shaded green (except the sushi-domain specific cysteine) and orange, as are identical matching residues in the other aligned sequences. In human IL-2R $\alpha$, the first and second sushi-domains engage in a beta strand exchange event in which the first 18 residues depicted here are swapped with a stretch of domain 2; this $1-18$ region is thus not functionally comparable with the IL- $15 R \alpha$ fragments to which it is aligned here. The IL-2R $\alpha$ residues indicated by pound symbols (\#) interact with IL-2 residues that contribute to patch 1 (Rickert et al. 2005). For GenBank accession numbers, see Fig. $\mathrm{S} 1 F$

the IL-2R $\alpha / 15 \mathrm{R} \alpha$ molecules agrees with the findings that these residues interact with each other in both IL-2-IL-2R $\alpha$ and IL-15·IL-15R $\alpha$ complexes (Rickert et al. 2005; Olsen et al. 2007). Also notable is that IL-15 residue E54 interacts with IL-15R $\alpha$ residue R26 (Chirifu et al. 2007; Olsen et al. 2007), and that Figs. 3 and 5 and Fig. S6 $A$ and show that mammalian IL-2 and IL-2R $\alpha$ lack those residues, but that in IL- 2 and IL-2R $\alpha$ of several more primitive species including chicken those residues can be found. Thus, it will be interesting to investigate when in evolution IL-2 and IL-15 acquired specificity for their individual receptor chains IL-2R $\alpha$ and IL$15 R \alpha$. In Text S1.2 we speculate about that development, also in regard to cis - versus trans-presentation and the association of IL-2 with Treg function.

\section{Conclusion and future prospects}

IL-2 and IL-15 are among the most potent and best studied cytokines, and it is surprising that with $I L-15 L$ a gene for an unknown mammalian family member could be detected. Although in many eutherian mammals the $I L-15 L$ ORF was incapacitated, in the most important agricultural mammals intact $I L-15 L$ was found, and rates of synonymous versus non-synonymous nucleotide exchanges do suggest preservation of protein function. Our continuing lines of research involve a search for endogenous IL-15L protein, and production of stable recombinant IL-15L protein, the latter which currently proves difficult (not shown). After IL-15L function 
will be known we can speculate about possible use in veterinary medicine. Hopefully and importantly, future understanding of IL-15L-IL-15R $\alpha$ structure may inspire widening of the range of agonists and antagonists for regulating IL-15 pathways in human medicine (e.g., Bernard et al. 2004; Zhu et al. 2009). Clarification of the evolution of the IL-2/15/15L family may also help to understand medically relevant mechanisms deciding between immune tolerance, in which IL-2 plays an important role, and inflammation mediated by IL-15. In summary, the intriguing conclusion of our study is that the mammalian IL-2 and IL-15 pathways developed in the presence of another IL-15R $\alpha$ binding molecule, namely IL-15L. And, furthermore, that motifs for cytokine with sushi-domain receptor chain interaction were very well conserved despite enormous diversification of the overall cytokine sequences, with the notable exception of the IL-2 system in the mammalian lineage which partially acquired a new and unique cytokine-to-receptor binding mode.

\section{Materials and methods}

\section{General}

Details and additional materials and methods are described in Text S1.1.

\section{Database searching and genetic software analysis}

Sequence databases at the National Center for Biotechnology Information (NCBI; http://www.ncbi.nlm.nih.gov/), Emsembl (http://www.ensembl.org/index.html) and the Elephant Shark Genome Project (http://esharkgenome.imcb.a-star.edu.sg/) were screened for $I L-2, I L-15, I L-15 L, I L-2 R \alpha$ and $I L-15 R \alpha$. Leader peptides were predicted by SignalP software (http:// www.cbs.dtu.dk/services/SignalP/) and alpha-helices were predicted by Phyre software (http://www.sbg.bio.ic.ac.uk/ phyre/).

\section{Analysis by fluorescence-activated cell sorting (FACS)} of transfected cells

HEK293 cells were transfected with plasmids described in Fig. S6, and 2 days after transfection half of the cells were fixed with $4 \%$ paraformaldehyde, followed by permeabilization with $0.01 \%$ digitonin. Both unfixed and fixed/ permeabilized cells were subsequently stained with the murine monoclonal antibodies "ANTI-FLAG ${ }^{\circledR}$ M2" (Sigma) or "MAb to C-myc" (Meridian Life Science), washed, and then incubated with Alexa Fluor ${ }^{\circledR} 488 \mathrm{~F}\left(\mathrm{ab}^{\prime \prime}\right) 2$ fragment of goat anti-mouse $\operatorname{IgG}(\mathrm{H}+\mathrm{L})$ (Fisher Scientific). After a final washing step, cells were resuspended with FACS buffer containing propidium iodide (PI) and analyzed with a BD
FACSCalibur flow cytometer (Becton Dickinson). Nonfixed PI-negative cells were regarded as live cells.

Acknowledgement Our gratitude goes to Dr. Karin Wiebauer, Senior freelance molecular- and computer biologist, Germany, for bringing the existence of $I L-15 L$ gene sequences in the mammalian genome to our attention. Dr. Wiebauer correctly predicted the structures of the mammalian $I L-15 L$ genes far beyond the obvious and contributed in numerous discussions to other topics of this study.

Open Access This article is distributed under the terms of the Creative Commons Attribution License which permits any use, distribution, and reproduction in any medium, provided the original author(s) and the source are credited.

\section{References}

Almeida AR, Legrand N, Papiernik M, Freitas AA (2002) Homeostasis of peripheral CD4+ T cells: IL-2R $\alpha$ and IL-2 shape a population of regulatory cells that controls $\mathrm{CD} 4+\mathrm{T}$ cell numbers. J Immunol 169(9):4850-4860

Anderson DM et al (1995) Functional characterization of the human interleukin-15 receptor alpha chain and close linkage of IL15RA and IL2RA genes. J Biol Chem 270(50):29862-29869

Bei JX, Suetake H, Araki K, Kikuchi K, Yoshiura Y, Lin HR, Suzuki Y (2006) Two interleukin (IL)-15 homologues in fish from two distinct origins. Mol Immunol 43(7):860-869

Bergamaschi C, Rosati M, Jalah R, Valentin A, Kulkarni V, Alicea C, Zhang GM, Patel V, Felber BK, Pavlakis GN (2008) Intracellular interaction of interleukin- 15 with its receptor alpha during production leads to mutual stabilization and increased bioactivity. J Biol Chem 283(7):4189-4199

Bergamaschi C, Bear J, Rosati M, Beach RK, Alicea C, Sowder R, Chertova E, Rosenberg SA, Felber BK, Pavlakis GN (2012) Circulating interleukin-15 (IL-15) exists as heterodimeric complex with soluble IL-15 receptor alpha (IL-15R $\alpha$ ) in human serum. Blood 120(1):e1-8

Bernard J et al (2004) Identification of an interleukin-15alpha receptorbinding site on human interleukin-15. J Biol Chem 279(23):2431324322

Bird S, Zou J, Kono T, Sakai M, Dijkstra JM, Secombes C (2005) Characterisation and expression analysis of interleukin 2 (IL-2) and IL-21 homologues in the Japanese pufferfish, Fugu rubripes, following their discovery by synteny. Immunogenetics 56(12):909-923

Bondensgaard K, Breinholt J, Madsen D, Omkvist DH, Kang L, Worsaae A, Becker P, Schiødt CB, Hjorth SA (2007) The existence of multiple conformers of interleukin-21 directs engineering of a superpotent analogue. J Biol Chem 282(32):23326-23336

Chirifu M, Hayashi C, Nakamura T, Toma S, Shuto T, Kai H, Yamagata Y, Davis SJ, Ikemizu S (2007) Crystal structure of the IL-15-IL15Ralpha complex, a cytokine-receptor unit presented in trans. Nat Immunol 8(9):1001-1007

Choi KD, Lillehoj HS, Song KD, Han JY (1999) Molecular and functional characterization of chicken IL-15. Dev Comp Immunol 23(2): 165-177

Donnelly RP, Sheikh F, Dickensheets H, Savan R, Young HA, Walter MR (2010) Interleukin-26: an IL-10-related cytokine produced by Th17 cells. Cytokine Growth Factor Rev 21(5):393-401

Dubois S, Mariner J, Waldmann TA, Tagaya Y (2002) IL-15Ralpha recycles and presents IL-15 In trans to neighboring cells. Immunity 17(5):537-547 
Fang W, Xiang LX, Shao JZ, Wen Y, Chen SY (2006) Identification and characterization of an interleukin-15 homologue from Tetraodon nigroviridis. Comp Biochem Physiol B Biochem Mol Biol 143(3): 335-343

Fang W, Shao JZ, Xiang LX (2007) Molecular cloning and characterization of IL-15R alpha gene in rainbow trout (Oncorhynchus mykiss). Fish Shellfish Immunol 23(1):119-127

Fontenot JD, Rasmussen JP, Gavin M-A, Rudensky AY (2005) A function for interleukin 2 in Foxp3-expressing regulatory $\mathrm{T}$ cells. Nat Immunol 6(11):1142-1151

Giri JG, Ahdieh M, Eisenman J, Shanebeck K, Grabstein K, Kumaki S, Namen A, Park LS, Cosman D, Anderson D (1994) Utilization of the beta and gamma chains of the IL-2 receptor by the novel cytokine IL-15. EMBO J 13(12):2822-2830

Giri J-G, Kumaki S, Ahdieh M, Friend DJ, Loomis A, Shanebeck K, DuBose R, Cosman D, Park LS, Anderson DM (1995) Identification and cloning of a novel IL-15 binding protein that is structurally related to the alpha chain of the IL-2 receptor. EMBO J 14(15): 3654-3663

Grabstein KH et al (1994) Cloning of a T cell growth factor that interacts with the beta chain of the interleukin-2 receptor. Science 264(5161): 965-968

Gunimaladevi I, Savan R, Sato K, Yamaguchi R, Sakai M (2007) Characterization of an interleukin-15 like (IL-15L) gene from zebrafish (Danio rerio). Fish Shellfish Immunol 22(4):351-362

Hage T, Sebald W, Reinemer P (1999) Crystal structure of the interleukin4/receptor alpha chain complex reveals a mosaic binding interface. Cell 97(2):271-281

Hamming OJ, Kang L, Svensson A, Karlsen JL, Rahbek-Nielsen $\mathrm{H}$, Paludan SR, Hjorth SA, Bondensgaard K, Hartmann R (2012) Crystal structure of interleukin-21 receptor (IL-21R) bound to IL-21 reveals that sugar chain interacting with WSXWS motif is integral part of IL-21R. J Biol Chem 287(12):9454-9460

Huising MO, Kruiswijk CP, Flik G (2006) Phylogeny and evolution of class-I helical cytokines. J Endocrinol 189(1):1-25

Kaiser P, Mariani P (1999) Promoter sequence, exon:intron structure, and synteny of genetic location show that a chicken cytokine with T-cell proliferative activity is IL2 and not IL15. Immunogenetics 49(1): 26-35

Kennedy MK et al (2000) Reversible defects in natural killer and memory CD8 T cell lineages in interleukin 15-deficient mice. J Exp Med 191(5):771-780

Kono T, Bird S, Sonoda K, Savan R, Secombes CJ, Sakai M (2008) Characterization and expression analysis of an interleukin-7 homologue in the Japanese pufferfish, Takifugu rubripes. FEBS J 275(6): $1213-1226$

Leonard WJ (2008) Type I cytokines and interferons and their receptors. In: Paul WE (ed) Fundamental immunology, 6th edn. Lippincott Williams \& Wilkins, Philadelphia, pp 706-749

Levin AM et al (2012) Exploiting a natural conformational switch to engineer an interleukin-2 'superkine'. Nature 484(7395):529-533

Liao W, Lin JX, Leonard WJ (2013) Interleukin-2 at the crossroads of effector responses, tolerance, and immunotherapy. Immunity 38(1):13-25

Malek T-R (2008) The biology of interleukin-2. Annu Rev Immunol 26: 453-479

Meredith RW et al (2011) Impacts of the Cretaceous Terrestrial Revolution and $\mathrm{KPg}$ extinction on mammal diversification. Science 334(6055):521-534

Mortier E, Quéméner A, Vusio P, Lorenzen I, Boublik Y, Grötzinger J, Plet A, Jacques Y (2006) Soluble interleukin-15 receptor alpha (IL$15 \mathrm{R}$ alpha)-sushi as a selective and potent agonist of IL-15 action through IL-15R beta/gamma. Hyperagonist IL-15 x IL-15R alpha fusion proteins. J Biol Chem 281(3):1612-1619

Mortier E, Woo T, Advincula R, Gozalo S, Ma A (2008) IL-15Ralpha chaperones IL-15 to stable dendritic cell membrane complexes that activate NK cells via trans presentation. J Exp Med 205(5):12131225

Myszka DG, Arulanantham PR, Sana T, Wu Z, Morton TA, Ciardelli TL (1996) Kinetic analysis of ligand binding to interleukin-2 receptor complexes created on an optical biosensor surface. Protein Sci 5(2): $2468-2478$

Nomiyama $\mathrm{H}$ et al (2008) Extensive expansion and diversification of the chemokine gene family in zebrafish: identification of a novel chemokine subfamily CX. BMC Genomics 9:222

Ohtani M, Hayashi N, Hashimoto K, Nakanishi T, Dijkstra JM (2008) Comprehensive clarification of two paralogous interleukin 4/13 loci in teleost fish. Immunogenetics 60(7):383-397

Olsen SK et al (2007) Crystal Structure of the interleukin-15.interleukin15 receptor alpha complex: insights into trans and cis presentation. $\mathrm{J}$ Biol Chem 282(51):37191-37204

Parrish-Novak J, Foster DC, Holly RD, Clegg CH (2002) Interleukin-21 and the IL-21 receptor: novel effectors of NK and T cell responses. J Leukoc Biol 72(5):856-863

Rickert M, Wang X, Boulanger MJ, Goriatcheva N, Garcia KC (2005) The structure of interleukin-2 complexed with its alpha receptor. Science 308(5727): 1477-1480

Ring AM et al (2012) Mechanistic and structural insight into the functional dichotomy between IL-2 and IL-15. Nat Immunol 13(12):1187-1195

Rozwarski DA, Gronenborn AM, Clore GM, Bazan JF, Bohm A, Wlodawer A, Hatada M, Karplus PA (1994) Structural comparisons among the short-chain helical cytokines. Structure 2(3):159-173

Sadlack B, Löhler J, Schorle H, Klebb G, Haber H, Sickel E, Noelle RJ, Horak I (1995) Generalized autoimmune disease in interleukin-2deficient mice is triggered by an uncontrolled activation and proliferation of CD4+ T cells. Eur J Immunol 25(11):3053-3059

Sandau MM, Schluns KS, Lefrancois L, Jameson SC (2004) Cutting edge: transpresentation of IL-15 by bone marrow-derived cells necessitates expression of IL-15 and IL-15R alpha by the same cells. J Immunol 173(11):6537-6541

Secombes CJ, Wang T, Bird S (2011) The interleukins of fish. Dev Comp Immunol 35(12):1336-1345

Shakhsi-Niaei M, Drögemüller M, Jagannathan V, Gerber V, Leeb T (2013) IL26 gene inactivation in Equidae. Anim Genet Epub ahead of print

Stauber DJ, Debler EW, Horton PA, Smith KA, Wilson IA (2006) Crystal structure of the IL-2 signaling complex: paradigm for a heterotrimeric cytokine receptor. Proc Natl Acad Sci USA 103(8):2788-2793

Sugamura K, Asao H, Kondo M, Tanaka N, Ishii N, Ohbo K, Nakamura M, Takeshita T (1996) The interleukin-2 receptor gamma chain: its role in the multiple cytokine receptor complexes and $\mathrm{T}$ cell development in XSCID. Annu Rev Immunol 14:179-205

Sundick RS, GillDixon C (1997) A cloned chicken lymphokine homologous to both mammalian IL-2 and IL-15. J Immunol 159(2):720-725

Taniguchi T, Matsui H, Fujita T, Takaoka C, Kashima N, Yoshimoto R, Hamuro J (1983) Structure and expression of a cloned cDNA for human interleukin-2. Nature 302(5906):305-310

Van de Peer Y, Frickey T, Taylor J, Meyer A (2002) Dealing with saturation at the amino acid level: a case study based on anciently duplicated zebrafish genes. Gene 295(2):205-211

Vincent M, Bessard A, Cochonneau D, Teppaz G, Solé V, Maillasson M, Birklé S, Garrigue-Antar L, Quéméner A, Jacques Y (2013) Tumor targeting of the IL-15 superagonist RLI by an anti-GD2 antibody strongly enhances its antitumor potency. Int J Cancer 133(3):757-765

Waldmann TA, Tagaya Y (1999) The multifaceted regulation of interleukin-15 expression and the role of this cytokine in NK cell differentiation and host response to intracellular pathogens. Annu Rev Immunol 17:19-49

Waldmann TA (2006) The biology of interleukin-2 and interleukin-15: implications for cancer therapy and vaccine design. Nat Rev Immunol 6(8):595-601

Wang X, Rickert M, Garcia KC (2005) Structure of the quaternary 
complex of interleukin-2 with its alpha, beta, and gammac receptors. Science 310(5751):1159-1163

Wang T, Holland J-W, Carrington A, Zou J, Secombes C-J (2007) Molecular and functional characterization of IL-15 in rainbow trout Oncorhynchus mykiss: a potent inducer of IFN-gamma expression in spleen leukocytes. J Immunol 179(3):1475-1488

Wang D, Deng X, Leng X, Mao X (2010) Interleukin-15 receptordirected immunotoxins attenuate disease severity in rat adjuvant arthritis. Mol Immunol 47(7-8):1535-1543
Wen Y, Fang W, Xiang LX, Pan RL, Shao JZ (2011) Identification of Treg-like cells in Tetraodon: insight into the origin of regulatory $\mathrm{T}$ subsets during early vertebrate evolution. Cell Mol Life Sci 68(15): 2615-2626

Xu L, Yang L, Liu W (2013) Distinct evolution process among type I interferon in mammals. Protein Cell 4(5):383-392

Zhu X, Marcus WD, Xu W, Lee HI, Han K, Egan JO, Yovandich JL, Rhode PR, Wong HC (2009) Novel human interleukin-15 agonists. J Immunol 183(6):3598-3607 